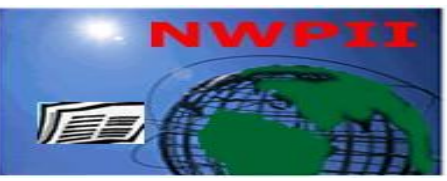

American Journal of Biomedical Sciences

ISSN: 1937-9080

nwpii.com/ajbms

\title{
Quality of Life of People Living with Type 2 Diabetes in Ho, Ghana: A Cross-Sectional Study
}

\author{
James Osei-Yeboah $^{{ }^{*}}$, William K. B. A. Owiredu ${ }^{2,5}$, Gameli Kwame Norgbe ${ }^{3}$, Sylvester Yao Lokpo ${ }^{1}$, \\ Emmanuel Alote Allotey ${ }^{1}$, Edinam Abla Doe ${ }^{1}$ and Florence A. Attah ${ }^{4}$
}

${ }^{1}$ Department of Medical Laboratory Sciences, School of Allied Health Sciences, University of Health and Allied Sciences, Ho, Ghana.

${ }^{2}$ Department of Molecular Medicine, School of Medical Sciences, Kwame Nkrumah University of Science and Technology, Kumasi, Ghana.

${ }^{3}$ School of Allied Health Sciences, University of Health and Allied Sciences, Ho, Ghana.

${ }^{4}$ Diabetic Clinic Ho Municipal Hospital, Ghana Health Service Ho, Volta Region, Ghana.

${ }^{5}$ Department of Clinical Biochemistry, Diagnostic Directorate, Komfo Anokye Teaching Hospital, Kumasi, Ghana.

"Corresponding Author

James Osei-Yeboah

Department of Medical Laboratory Sciences

School of Allied Health Sciences

University of Health and Allied Sciences

Ho, Ghana.

Email: joyeboah@uhas.edu.gh

Received: 08 Auguest 2016; | Revised: 08 December 2016; | Accepted: 14 December 2016

\begin{abstract}
Background: Health related quality of life represents the effect of an illness on a patient, as perceived by the patient, and yields complementary information used as an outcome measurement. This study evaluated the quality of life of patients with diabetes mellitus and its relationship with clinical laboratory endpoint parameters. Methods: A hospital-based cross-sectional study was conducted between January, 2016 and April 2016. One hundred and fifty (150) diabetic patients attending diabetic clinic at the Ho Municipal Hospital were recruited for this study. Using a semi-structured instrument, data including clinical, socio-demography and quality of life was captured. Serum creatinine and fasting blood glucose were estimated for all the respondents. Results: Among the total study population, diabetes affected the quality of life of $12 \%$ severely and majority (52\%) moderately. Risk of anxiety and worry as well as sexual dysfunction was associated with the male gender. Increasing renal insufficiency was observed to degrade the overall quality of life of a diabetic patient. Conclusion: People living with diabetes suffer from deteriorating quality of life. The severity however is influenced by gender and clinical endpoints such as renal insufficiency and complications.
\end{abstract}

Keywords: Health Related Quality of Life, Diabetes, Chronic Kidney Disease, Diabetes 39, Clinical endpoint 


\section{Background}

The surge in the prevalence of diabetes mellitus in both the developed and developing countries, has made diabetes one of the global public health threats [1]. Type 2 diabetes is said to account for majority of cases of the disease ( $85 \%$ to $95 \%$ ) in low income countries [2].

In Ghana, it is estimated that 4 million people are living with diabetes and this number is expected to rise in the near future [3]. Patients living with diabetes are at risk of complications from the disease including loss of blood circulation to the heart and limbs, risk of developing multiple microvascular and cardiovascular complications, retinopathy, reduced life-expectancy, stroke, hypertension, nephropathy, neuropathy, impotence, skin lesion and musculoskeletal problems $[4,5]$. These complications further pose great mental and social challenges to the individuals as they have to adjust to the experiences of living with diabetes [3] . Diabetes thus represents one of the most important chronic diseases in the population, with regards to its impact on health [6]. Diabetes and its management can have a considerable impact on people's lives for example, feelings of isolation, co-dependency, experience of loss, overuse of defence mechanisms, and loss of freedom, all of which could have consequences for the optimal management of the condition [7].

Quality of life is a concept that covers a broad range of human experience and in the medical domain it denominates aspects of the health from the patient's or subject's point of view, and could better be expressed as "subjective health" or "functional status and wellbeing"[6]. Health related quality of life thus represents the effect of an illness on a patient, as perceived by the patient, and yields complementary information to medical or epidemiological data which may be used as an outcome measurement [8]. It is therefore characterised as "the ultimate goal of all health interventions [6].

Due to insufficiency of traditional end points (which are mainly focused on the biologic and physiologic outcomes) in capturing the effects of interventions on patients' health-related quality of life (HRQoL), a growing interest has emerged during the past decades for assessing determinant factors of patients' HRQoL, especially in chronic diseases like diabetes [9]. Diabetes is a chronic disease with considerable impact on health status and quality of life [10]. Several studies have demonstrated that diabetes has a strong negative impact on the health-related quality of life (HRQoL), especially in the presence of complications $[1,7,11]$.

Among the few studies that related traditional clinical end points to patient-centered outcomes (HRQoL) among diabetes patients, Khanna, Bush [8] reported that improvements in haemoglobin $\mathrm{A} 1_{\mathrm{C}}(\mathrm{HbA} 1 \mathrm{c})$ among diabetics was associated with better diabetes-specific quality of life. There is dearth of information on the quality of life of people living with diabetes in the Ghanaian setting and the few found in the literature [12] did not relate quality of life with clinical end-point parameters. This study therefore aims to investigate the quality of life of patients with diabetes mellitus and its relationship with laboratory data as clinical endpoint parameters.

\section{Materials and methods}

\subsection{Study Population and Design}

A hospital-based cross-sectional study was conducted between January and April 2016. One hundred and fifty (150) participants were involved in this study. These were diabetic patients under clinical management at the diabetic clinic of the Ho Municipal Hospital who subscribed to the National Health Insurance Scheme. The study participants were recruited purposefully from a population of adult individuals between the ages of $18-87$ years. The criteria for participation were patients who attended the diabetes clinic at the Municipal Hospital (outpatients) whose laboratory results had been captured into the hospital's records.

\subsection{Socio-demographic and HQoL data capture (Questionnaire)}


A self-reported semi-structured questionnaire was administered to determine the marital status, duration of diabetes, educational level, religion, occupation or employment status, medication type, and complications et cetera. using the prevalidated seven (7) point Likert scale, Diabetes 39 instrument, patients self-assessment of the extent to which diabetes affected their life was assessed [13].

\subsection{Blood sample collection and test procedure}

Following standard phlebotomy procedures about three $3 \mathrm{mls}$ of venous blood was collected from the antecubital fossa of the study participants after an overnight fast (12-16 hours) between $7 \mathrm{am}$ and $10 \mathrm{am}$ and dispensed into a serum separator tube. The samples were centrifuged at 3000rpm (revolutions per minute) for 5 minutes and the serum separated. Assay parameters included: fasting blood Glucose and Creatinine. The methods adopted for the determination of the Glucose and Creatinine were predetermined by the reagent manufacturer (ELITech Clinical Systems, SAS, Zone Indusrrielle-61500 SEES, France) and assays were performed using the selectra junior Chemistry autoanalyzer (Vital Scientific, B.V).

\subsection{Diagnosis of Chronic Kidney Disease (CKD)}

Glomerular filtration rate was calculated using the Four Variable Modification of Diet in Renal Disease -4v-MDRD and the Chronic Kidney Disease Epidemiology CollaborationCKD-EPI equations. The estimated glomerular filtration rate (eGFR) results from the two renal function equations were used to stratify the study population into five categories corresponding with the five stages of CKD in the K/DOQI CKD classification [14]. Chronic kidney disease was classified as eGFR $<60 \mathrm{~mL} / \mathrm{min} / 1.73 \mathrm{~m}^{2}$ (Stage 3,4 and 5, see Table 4) [15]

\subsubsection{Four Variable Modification of Diet in Renal Disease (4v-MDRD)}

4v - MDRD: eGFR $=$

$186 \mathrm{X} \mathrm{SCr}^{-1.154} \mathrm{X} \mathrm{Age}^{-0.203} \mathrm{X}(1.212) \mathrm{X}(0.742$ if female $)$

\subsubsection{Chronic Kidney Disease Epidemiology collaboration (CKD-EPI)}

\begin{tabular}{|l|l|l|}
\hline Gender & $\begin{array}{l}\text { Serum Creatinine } \\
\boldsymbol{\mu m o l} / \mathbf{L}(\mathbf{m g} / \mathbf{d L})\end{array}$ & Estimated Glomerular Equation \\
\hline Female & $\leq 62(\leq 0.7)$ & eGFR $=166 \times\left(\frac{\text { Serum Creatinine }}{0.7^{-0.329}}\right) \times(0.993)^{\mathrm{Age}}$ \\
\hline Female & $>62(>0.7)$ & $\mathrm{eGFR}=166 \times\left(\frac{\text { Serum Creatinine }}{0.7^{-1.209}}\right) \times(0.993)^{\mathrm{Age}}$ \\
\hline Male & $\leq 80(\leq 0.9)$ & eGFR $=163 \times\left(\frac{\text { Serum Creatinine }}{0.9^{-0.411}}\right) \times(0.993)^{\mathrm{Age}}$ \\
\hline Male & $>80(>0.9)$ & eGFR $=163 \times\left(\frac{\text { Serum Creatinine }}{0.9^{-1.209}}\right) \times(0.993)^{\mathrm{Age}}$ \\
\hline
\end{tabular}

\section{Data Analysis}


The sum of scores for each section, the total score and classifications of self-perceived quality of life was transformed to a scale from 0 to 100 with higher scores indicating greater severity of affected quality of life by applying the generic linear transformation formula [13].

$$
\frac{(\text { Gross classification }- \text { Minimum value })}{\text { (Maximum value }- \text { Minimum value) }} 100
$$

For the purpose of this study a score of 0 to 20 suggest quality of life not affected by diabetes, 21 to 40 moderately affected and a score greater than 40 poor/severely affected quality of life in accordance with the cut-off for cumulative percentage in Al Rubaish, Wosornu [16].

Categorical outcomes were expressed as frequency and percentage. Differences between proportions were tested with Chi square or Fishers Exact test where appropriate using GraphPad Prism version 6.00 GraphPad software, San Diego, California USA, (www.graphpad.com) for windows for statistical analysis

\section{Results}

As shown in Table 1, the study population comprised 150 respondents with $99(66 \%)$ being females. The age range of the study participants was from 18 to 87 years and majority of the respondents were above 40 years. Most of the respondents had attained at least basic level of education at the time of the study (89.33\%). Christianity was the dominant religion among the study population (94.00\%). Majority of the participants in this study were employed in the informal sector (44.67\%). The entire study population was on medication and the commonest clinical complication recorded among study participants was blurred vision (See table 1).

Among the general study population diabetes was found not to affect the quality of life of $36 \%$ of the study participants, but however, moderately affected $52 \%$ and severely affected the quality of life of $12 \%$. The stratification of recorded quality of life scores by the sociodemographic characteristics of people living with diabetes as seen from table 2, revealed that respondent age, gender, religion and occupation had no significant influence on the quality of life. However, patients' attained level of formal education significantly distinguished patients' quality of life. The highest percentage of the group experiencing poor quality of life was found among patients with basic education (20.69\%) whereas those with no formal education presented with the least proportion of respondents with excellent quality of life (18.75\%) (See table 2).

Among the general study population, $12 \%$ were classified as having poor (severely affected) overall health related quality of life due to diabetes. Anxiety and worry $(23.33 \%)$ and affected sexual function $(22.67 \%)$ were the most prevalent of low/poor (severely affected) quality of life component domains among people living with diabetes in the Ho municipality. The levels at which diabetes affects the different facets of life among patients revealed no significant difference between the male and the female participants in the overall quality of life as well as the energy and mobility domain, diabetes control domain and the social overload domain. Significant gender variations was exhibited in the domains that bordered on anxiety and worry and sexual function; with significantly higher proportions of the male participants presenting with anxiety and worry $19(37.25 \%)$ and problems with sexual function 28 (54.90\%) (See Table 3).

Among the total population of 150 participants, $12.00 \%$ presented with chronic kidney disease (CKD) when assessed by the four variable Modification of Diet in Renal Disease (4v-MDRD) equation. Using the Chronic Kidney Disease Epidemiology Collaboration equation (CKD-EPI) in the estimation of the glomerular filtration rate (eGFR), 19 people representing $12.67 \%$ of the study participants presented with CKD. As shown in table 4, irrespective of the kidney function equation used all patients presenting with CKD were in stage three (eGFR of $30-59 \mathrm{~mL} / \mathrm{min} / 1.73 \mathrm{~m}^{2}$ ). 
Table 1. Socio-demographics characteristics of people living with diabetes attending clinic at the Ho Municipal Hospital.

\begin{tabular}{|c|c|c|c|}
\hline Parameters & Total & Female & Male \\
\hline Total Respondents & $150(100)$ & $99(66.00 \%)$ & $51(34.00 \%)$ \\
\hline \multicolumn{4}{|l|}{ Age } \\
\hline$\leq 40$ years & $8(5.33)$ & $6(6.06)$ & $2(3.92)$ \\
\hline$>40$ years & 142(94.67) & 93(93.94) & $49(96.08)$ \\
\hline \multicolumn{4}{|l|}{ Marital Status } \\
\hline Cohabitation & $2(1.33)$ & $2(2.02)$ & $0(0.00)$ \\
\hline Married & $100(66.67)$ & $57(57.58)$ & $43(84.31)$ \\
\hline Single & $48(32.00)$ & $40(40.40)$ & $8(15.69)$ \\
\hline \multicolumn{4}{|l|}{ Educational Level } \\
\hline None & $16(10.67)$ & $16(16.16)$ & $0(0.00)$ \\
\hline Basic & $58(38.67)$ & $43(43.43)$ & $15(29.41)$ \\
\hline Secondary & $38(25.33)$ & $25(25.25)$ & $13(25.49)$ \\
\hline Tertiary & $38(25.33)$ & $15(15.15)$ & $23(45.10)$ \\
\hline \multicolumn{4}{|l|}{ Religion } \\
\hline Christian & $141(94.00)$ & $93(93.94)$ & $48(94.12)$ \\
\hline Muslim & $6(4.00)$ & $4(4.04)$ & $2(3.92)$ \\
\hline Traditionalist & $3(2.00)$ & $2(2.02)$ & $1(1.96)$ \\
\hline \multicolumn{4}{|l|}{ Employment Status } \\
\hline None & $54(36.00)$ & $37(37.37)$ & $17(33.33)$ \\
\hline Formal & 29(19.33) & $14(14.14)$ & $15(29.41)$ \\
\hline Informal & $67(44.67)$ & $48(48.48)$ & $19(37.25)$ \\
\hline \multicolumn{4}{|l|}{ Medication } \\
\hline Drugs & 142(94.67) & $92(92.93)$ & $50(98.04)$ \\
\hline Insulin Injection & $8(5.33)$ & $7(7.07)$ & $1(1.96)$ \\
\hline \multicolumn{4}{|l|}{ Complications } \\
\hline None & $53(35.33)$ & $35(35.36)$ & $18(35.29)$ \\
\hline Blurred vision & $43(28.67)$ & $30(30.30)$ & $13(25.49)$ \\
\hline Hypertension & $33(22.00)$ & $20(20.20)$ & $13(25.49)$ \\
\hline Numbness & $21(14.00)$ & $14(14.14)$ & $7(13.73)$ \\
\hline
\end{tabular}

Data is presented as figure with corresponding percentages in parenthesis. 
Table 2. Quality of life of people living with diabetes stratified by socio- demographic characteristics of respondents

\begin{tabular}{|c|c|c|c|c|}
\hline Parameter & $\begin{array}{l}\text { Not Affected } \\
\quad(0 \text { to 20) }\end{array}$ & $\begin{array}{c}\text { Moderately } \\
\text { Affected } \\
\text { (21 to 40) }\end{array}$ & $\begin{array}{c}\text { Severely } \\
\text { Affected } \\
(>40)\end{array}$ & p-value \\
\hline Total Respondent & $54(36.00)$ & $78(52.00)$ & $18(12.00)$ & \multirow[b]{3}{*}{0.793} \\
\hline \multicolumn{5}{|l|}{ Age } \\
\hline$\leq 40$ & $2(25.00)$ & $5(62.50)$ & $1(12.50)$ & \\
\hline$>40$ & $52(36.62)$ & $73(51.41)$ & $17(11.97)$ & \\
\hline \multicolumn{5}{|l|}{ Gender } \\
\hline Female & $38(38.38)$ & $52(52.53)$ & $9(9.09)$ & \multirow[t]{2}{*}{0.282} \\
\hline Male & $16(31.37)$ & $26(50.98)$ & $9(17.65)$ & \\
\hline \multicolumn{5}{|l|}{ Religion } \\
\hline Christian & $49(34.75)$ & $74(52.48)$ & $18(12.77)$ & \multirow[t]{3}{*}{0.168} \\
\hline Muslim & $2(33.33)$ & $3(50.00)$ & $1(16.67)$ & \\
\hline Traditionalist & $3(100)$ & $0(0.00)$ & $0(0.00)$ & \\
\hline \multicolumn{5}{|l|}{ Educational status } \\
\hline None & $3(18.75)$ & $13(81.25)$ & $0(0.00)$ & \multirow[t]{3}{*}{0.023} \\
\hline Basic & $20(34.48)$ & $26(44.83)$ & $12(20.69)$ & \\
\hline Secondary & $12(31.58)$ & $23(60.53)$ & $3(7.89)$ & \\
\hline Tertiary & $19(50.00)$ & $16(42.11)$ & $3(7.89)$ & \multirow{4}{*}{0.382} \\
\hline \multicolumn{5}{|l|}{ Occupation } \\
\hline Formal & $14(48.28)$ & $13(44.83)$ & $2(6.90)$ & \\
\hline Informal & $19(28.36)$ & $38(56.72)$ & $10(14.93)$ & \\
\hline None & 21(38.89) & $27(50.00)$ & $6(11.11)$ & \\
\hline
\end{tabular}

Data is presented as figure and percentage, $\mathrm{p}$ is significant at 0.05 . 
Table 3. Gender distribution of poor quality of life (severely affected QoL >40) among diabetic patients attending clinic at the Ho Municipal Hospital

\begin{tabular}{lcccc}
\hline Parameter & Total & Male (51) & Female (99) & p-value \\
\hline Energy and Mobility & $25(16.67)$ & $8(15.69)$ & $17(17.17)$ & 1 \\
Diabetes Control & $19(12.67)$ & $7(13.73)$ & $12(12.12)$ & 0.7990 \\
Anxiety and Worry & $35(23.33)$ & $19(37.25)$ & $16(16.16)$ & 0.0074 \\
Sexual Function & $34(22.67)$ & $28(54.90)$ & $6(6.06)$ & $<0.0001$ \\
Social Overload & $15(10.00)$ & $8(15.69)$ & $7(7.07)$ & 0.1481 \\
\hline Overall QoL & $18(12.00)$ & $9(17.65)$ & $9(9.09)$ & 0.1831 \\
\hline
\end{tabular}

Data is presented as figure and percentage, $\mathrm{p}$ is significant at 0.05 . QoL-Quality of life.

Table 4. Estimates of the prevalence of chronic kidney disease in the study population using the renal function equations

\begin{tabular}{lcc}
\hline Parameter & 4v-MDRD & CKD-EPI \\
\hline Stage $1(\geq \mathbf{9 0})$ & $52(34.67)$ & $46(30.66)$ \\
Stage $2(\mathbf{6 0}$ to $\mathbf{8 9})$ & $80(53.33)$ & $85(56.67)$ \\
Stage $3(\mathbf{3 0}$ to $\mathbf{5 9})$ & $18(12.00)$ & $19(12.67)$ \\
Stage $4(\mathbf{1 5}$ to $\mathbf{2 9})$ & $0.00(0.00)$ & $0.00(0.00)$ \\
Stage $\mathbf{5}(<\mathbf{1 5})$ & $0.00(0.00)$ & $0.00(0.00)$ \\
\hline CKD $($ Stage $\mathbf{3 + 4 + 5})$ & $18(12.00)$ & $19(12.67)$ \\
\hline
\end{tabular}

Data is presented as absolute values with corresponding percentage in parenthesis. 4v-MDRD - four variable Modification of Diet in Renal Disease, CKD-EPI - Chronic Kidney Disease Epidemiology collaboration, CKD Chronic Kidney Disease 
The diabetes control component of the quality of life score was found to positively correlate with all other component domains and the overall quality of life scores. Aging was associated with a decline in the energy and mobility component of the quality of life. Patient's current glucose level was found to be associated with social overload, diabetes control and the overall quality of life. As seen in table 5, increasing renal insufficiency was observed to decrease the overall quality of life of a diabetic patient and also influences sexual function, social overload, anxiety and worry and diabetes control (See Table 5).

Table 5. Pearson correlation of component Quality of life score and glycaemic and renal laboratory indices

\begin{tabular}{|c|c|c|c|c|c|c|c|c|c|c|c|}
\hline Parameter & SF & SOV & $\mathbf{A W}$ & DC & EM & Overall & G-Past 1 & $\begin{array}{c}\text { G- } \\
\text { Past } 2\end{array}$ & $\begin{array}{c}\text { G- } \\
\text { Present }\end{array}$ & $\begin{array}{c}\text { 4V- } \\
\text { MDRD }\end{array}$ & $\begin{array}{c}\text { CKD- } \\
\text { EPI }\end{array}$ \\
\hline Age & -0.03 & 0.01 & -0.1 & -0.06 & $.19^{*}$ & -0.03 & $-.33 * *$ & $\begin{array}{c}- \\
.31^{*} *\end{array}$ & $-.32 * *$ & $-.30 * *$ & -0.05 \\
\hline SF & & $.22 * *$ & 0.13 & $.23^{* *}$ & $.17 *$ & $.20^{*}$ & 0.14 & 0.08 & 0.05 & $-.28 * *$ & $-.33 * *$ \\
\hline SOV & & & $.60 * *$ & $.64 * *$ & $.53 * *$ & $.76^{* *}$ & 0.11 & 0.08 & $.21 * *$ & $-.28 * *$ & -0.18 \\
\hline $\mathbf{A W}$ & & & & $.65^{* *}$ & $.53 * *$ & $.59 * *$ & 0.05 & 0.07 & 0.11 & $-.26 * *$ & -0.09 \\
\hline DC & & & & & $.55^{* *}$ & $.63^{* *}$ & 0.11 & 0.06 & $.16^{*}$ & $-.25 *$ & -0.1 \\
\hline EM & & & & & & $.56^{* *}$ & 0.06 & 0.03 & 0.07 & -0.02 & -0.12 \\
\hline Overall & & & & & & & 0.13 & 0.07 & $.18^{*}$ & $-.20 *$ & -0.04 \\
\hline G-Past 1 & & & & & & & & $.58 * *$ & $.59 * *$ & 0.15 & 0.13 \\
\hline G-Past 2 & & & & & & & & & $.46^{* *}$ & 0.12 & -0.02 \\
\hline G-Present & & & & & & & & & & 0.13 & 0.02 \\
\hline
\end{tabular}

Data is presented as correlation coefficient. *p is significant at 0.05 , **p is significant at 0.01 . SF-Sexual Function, SOV-Social Overload, AW-Anxiety and Worry, DC-Diabetes Control, EM- Energy and Mobility, G-Past 1-Glucose Past 1, G-Past 2-Glucose Past 2, G-Present- Glucose Present.

As shown in figure 1, different complications reported by participants were found to significantly affect the overall quality of life and the social overload component of the individuals. In case of social overload respondents presenting cardiovascular diseases and stroke classified as others were the most affected $(33.3 \%)$, followed by those experiencing numbness (14.3\%) (Figure 1A). The patients who had no clinical complication presented with the least affected overall quality of life $(6.4 \%)$, whilst half of those reporting with complications of cardiovascular diseases and stroke were affected (figure 1B). 


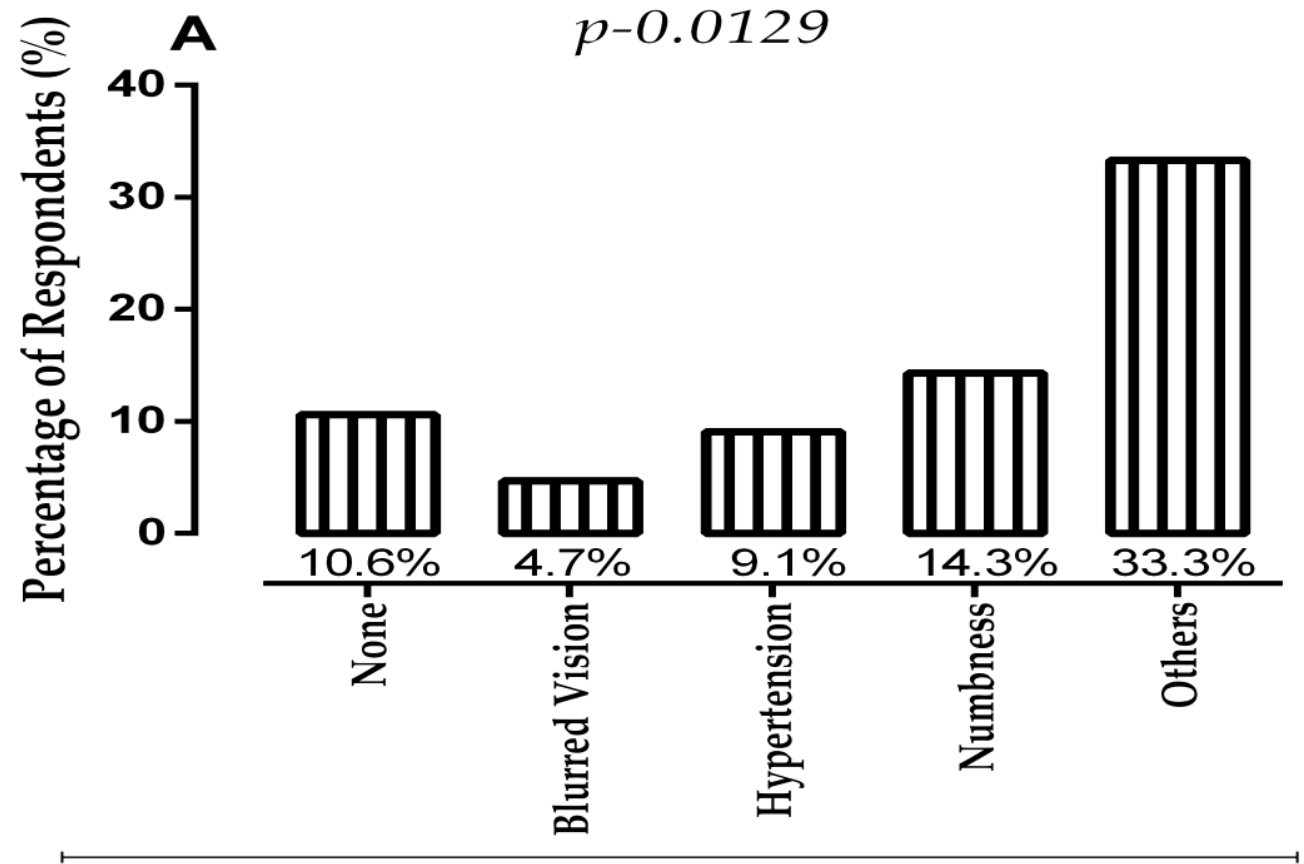

Social Overload Domain of Quality of Life

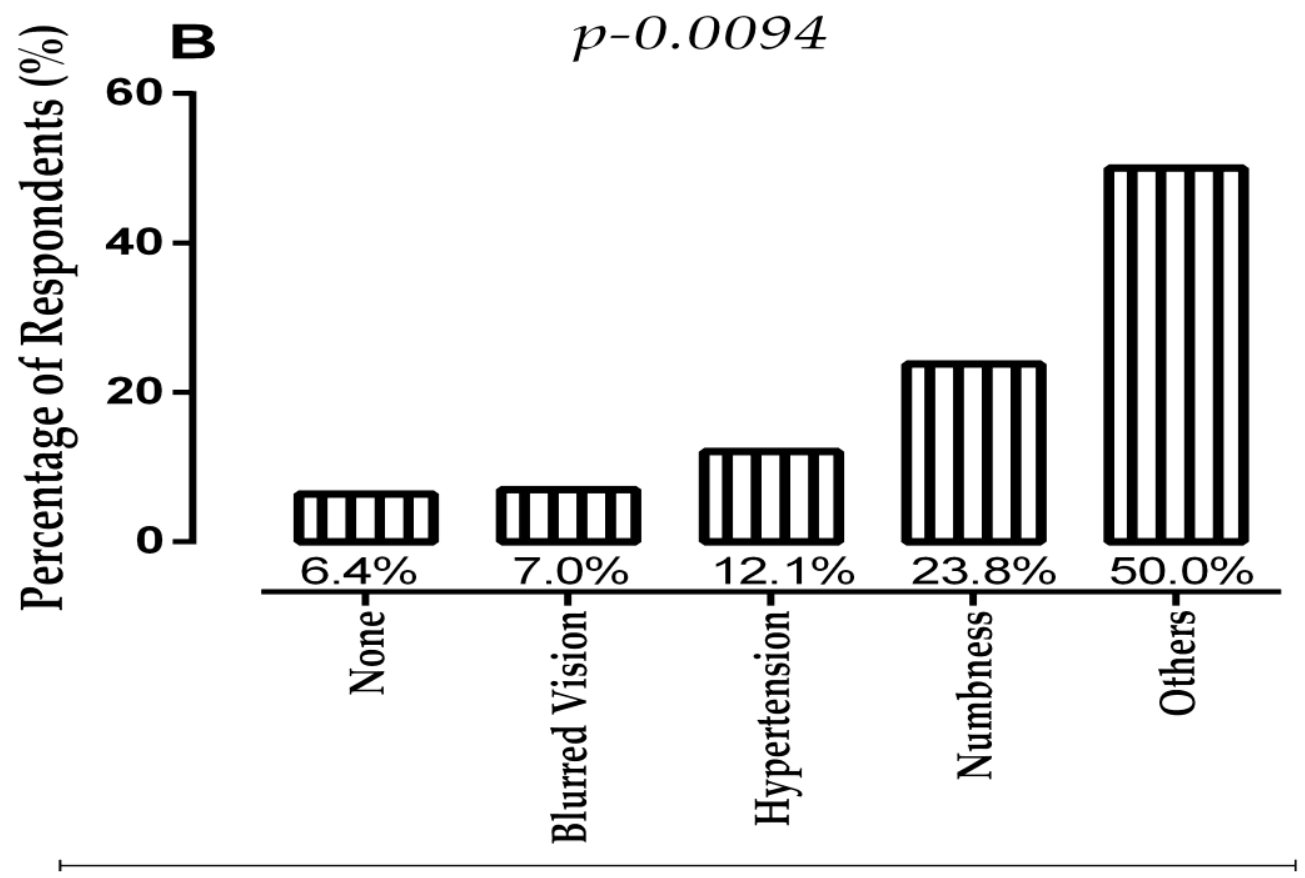

Overall Quality of Life

Figure 1. Clinical complication and quality of life of people living with diabetes. 


\section{Discussion}

Among the general study population, diabetes was found not to affect the quality of life of $36 \%$, moderately affect $52 \%$ and severely affect the quality of life of $12 \%$. Anxiety and worry $(23.33 \%)$ and affected sexual function (22.67\%) were the most prevalent of low/poor quality of life component domains among people living with diabetes in the Ho municipality (Table 2). In explaining the illness perception and mental problems among type 2 Ghanaian diabetes patients, Nyarko, Kugbey [3] posited that because diabetes is perceived as threatening among sufferers, it is likely to induce certain emotional feelings that are usually negative leading to the individual becoming depressed, anxious and psychologically distressed.

Majority of patients living with diabetes and seeking management at the Ho Municipal Hospital were females $99(66 \%)$ and were older than 40 years. This population is similar to that found by Danquah, Bedu-Addo [17] who characterized the population of people living with type 2 diabetes in urban Ghana.

Examination of the relationship of demographic factors to the diabetes quality of life (DQoL) measures suggests that they are not generally confounded by factors such as education, sex, or duration of diabetes [18]. In contrast, education was found to be the nondiabetic factor that confounded the overall quality of life among patients living with diabetes in the current study. The highest percentage of the group experiencing poor quality of life was found among patients who had attained basic education (20.69\%) whereas those with no formal education presented with the least proportion of respondents with no negative effect on quality of life $(18.75 \%)$. This finding is in concordance with the assertion that schooling is a highly significant predictor of quality of life $[19,20]$.

Gender variation was significantly exhibited in the component domains of anxiety and worry as well as sexual function with vulnerability tilted towards the males (Table 3). In other settings vulnerability to anxiety and other component domain of quality of life were observed to be significantly worse among females $[1,21,22]$.
Diabetic autonomic neuropathy is the most common complication of diabetes and among its major clinical manifestations is erectile dysfunction [23]. This may account for the high levels of affected sexual function among the male participants.

One of the most common complications of diabetes and the single strongest predictor of mortality in patients with diabetes is chronic kidney disease (CKD) $[24,25]$. In the current study the prevalence of CKD among the diabetic participants was $12.00 \%$ to $12.69 \%$ depending on the equation used in estimating the glomerular filtration rate (Table 4). Earlier reports by Amoako, Laryea [26] at the Komfo Anokye teaching hospital and Ephraim, Biekpe [27] at the Effia-Nkwanta regional and the Takoradi Government hospitals both recorded higher prevalence of CKD among diabetes patients $(22.2 \%$ and $27 \%$ respectively).

With the exception of energy and mobility domain, the overall quality of life as well as all the individual component domains were significantly affected negatively by increasing renal insufficiency (Table 5). According to Huang, Brown [28] and Solli, Stavem [29] endstage complications have the greatest perceived burden on quality of life of people living with diabetes. The findings of this study agrees with that of Parkerson Jr, Connis [30] who reported that patients with the diabetic complication of nephropathy had increased worry over their health and lower general health perceptions. Among a Ghanaian diabetes population Sarfo, Cudjoe [31] reported that the number of complications / clinical manifestations predict health-related quality of life (HRQoL).

In the presence of renal impairment in diabetes, both gluconeogenesis and glycogenolysis are impaired, yet there is insulin resistance [32]. Consequently, patients with diabetes and kidney disease are prone to hypoglycemia because the kidney cortices can provide up to half of gluconeogenesis in the postabsorptive state [32]. Mehta, Cull [5] reported that the fear of repeated episodes of hypoglycaemia among diabetic patients who had experienced this complication was a predictor of 
poorer health related quality of life among people living with diabetes.

In the present study patient's current glucose level was found to be associated with social overload, diabetes control and the overall quality of life. One of the pivotal factors that correlated significantly with diabetic general well-being is fasting blood glucose level [33]. Khanna, Bush [8] reported that improvements in HbA1c, a biochemical marker of glucose level among participants completing a diabetes selfmanagement program were associated with better diabetes-specific quality of life. The diabetes control component of the quality of life score was found to positively correlate with all other component domains and the overall quality of life scores (Table 5). Thus a person's perception on the control of his/her glucose level affected the quality of the other aspect of his/her life. Having better glycemic control is associated with better quality of life [34], but perceived lack of control of diabetes and the anticipation of worse consequences reduce the quality of life of the patient [35]. Thus the cognitive appraisal of the illness generates emotional, physiological and behavioral reactions that negatively influence the mental and physical health of the individual living with diabetes mellitus [3].

Diabetes is associated with both microvascular and macrovascular diseases affecting several organs [36]. In a review of health related quality of life among diabetic patients, the most consistently found and strongest predictors were macrovascular diseases, especially coronary heart disease, and nonvascular diseases [6]. In the present study different complications reported by participants were found to significantly affect the overall quality of life and the social overload component of the individuals. In agreement with Wändell [6], respondents presenting with cardiovascular diseases and stroke had significantly deteriorated social overload (33.3\%) and overall quality of life (Figure 1A \&1B)

\section{Conclusion}

Among the total study population diabetes affected the quality of life of $12 \%$ severely, and majority (52\%) moderately. Anxiety and worry were the most dominant components of health related quality of life affecting people living with diabetes. Being male was associated with the risk of anxiety and worry as well as affected sexual function. Increasing renal insufficiency was observed to decrease the overall quality of life of a diabetic patient and also affect negatively the aspect of sexual function, social overload, anxiety and worry and diabetes control. Thus the early clinical assessment and management of health related quality of life (HRQOL) in clinical practice could be beneficial among diabetics in our setting.

\section{Ethical Consideration}

Ethical approval for the study was granted by the facility authorized committee in a letter (HMH/G-207[Vol5]). The study was anonymous and non-linked, participation was voluntary and all patients consented to the study. Confidentiality of participant's information was assured and no patient was denied the appropriate care for declining to participate in this study.

\section{Competing Interests}

Authors have declared that no competing interests exist.

\section{Authors Contributions}

This work was carried out with collaboration between all authors. Authors JOY, WKBAO, GKN, SYL, EAA, EAD and FAA conceptualized and designed the study. Authors JOY, SYL, EAA, EAD and FAA recruited participants and generated the data. Authors JOY, WKBAO, GKN, SYL, EAA, EAD and FAA analyzed the data. Authors JOY, WKBAO, GKN, SYL, EAA, EAD and FAA drafted the manuscript. Authors JOY, WKBAO, GKN, SYL, EAA, EAD and FAA reviewed the manuscript for intellectual content and each author approved the final manuscript. 


\section{Acknowledgements}

We want to thank the management of the Ho Municipal Hospital for allowing us the permission to carry out this study in their facility. Our sincere gratitude goes to all Biomedical Scientists at the Ho Municipal Hospital, for without your participation this project would not have come into fruition.

\section{References}

1. Eljedi, A., R.T. Mikolajczyk, A. Kraemer, and U. Laaser, Health-related quality of life in diabetic patients and controls without diabetes in refugee camps in the Gaza strip: a cross-sectional study. $B M C$ Public Health, 2006. 6(1): p. 1. DOI: 10.1186/1471-2458-6-268

2. Hall, V., R.W. Thomsen, O. Henriksen, and N. Lohse, Diabetes in Sub Saharan Africa 1999-2011: Epidemiology and public health implications. a systematic review. BMC Public Health, 2011. 11(1): p. 1-12 DOI: $10.1186 / 1471-2458-11-564$.

3. Nyarko, K., N. Kugbey, and S. Atindanbila, Illness perception, religiosity and mental health of diabetic patients in Ghana. American Journal of Applied Psychology, 2014. 3(1): p. 12-20. DOI: $10.11648 /$ j.ajap.20140301.13

4. Darkwa, S., Prevalence of diabetes mellitus and resources available for its management in the Cape Coast Metropolis. ISABB Journal of Health and Environmental Sciences, 2011. 1(1): p. 17.

5. Mehta, Z., C. Cull, I. STratton, and J. Yudkin, Quality of life in type 2 diabetic patients is affected by complications but not by intensive policies to improve blood glucose or blood pressure control (UKPDS 37). Diabetes care, 1999. 22(7): p. 1125. DOI:10.2337/diacare.22.7.1125

6. Wändell, P.E., Quality of life of patients with diabetes mellitus An overview of research in primary health care in the Nordic countries. Scandinavian journal of primary health care, 2005. 23(2): p. 68-74.DOI: $10.1080 / 0281343 \quad 05100 \quad 15$ 296
7. Lindsay, G., K. Inverarity, and J.R. McDowell, Quality of life in people with type 2 diabetes in relation to deprivation, gender, and age in a new communitybased model of care. Nursing research and practice, 2011. 2011.DOI: 10.1155/2011/613589

8. Khanna, A., A.L. Bush, J.M. Swint, M.F. Peskin, R.L. Street, and A.D. Naik, Hemoglobin A 1c improvements and better diabetes-specific quality of life among participants completing diabetes self-management programs: A nested cohort study. Health and quality of life outcomes, 2012. 10(1): p. 1. DOI: 10.1186/1477-7525-10-48

9. Kiadaliri, A.A., B. Najafi, and $M$. Mirmalek-Sani, Quality of life in people with diabetes: a systematic review of studies in Iran. J Diabetes Metab Disord, 2013. 12(1): p. 54. DOI: 10.1186/22516581-12-54

10. Demirci, H., Y. Cinar, N. Bayram, and N. Bilgel, Quality of life in type II diabetic patients in primary health care. Dan Med J, 2012. 59(10): p. A4468.

11. Redekop, W.K., M.A. Koopmanschap, R.P. Stolk, G.E. Rutten, B.H. Wolffenbuttel, and L.W. Niessen, Healthrelated quality of life and treatment satisfaction in Dutch patients with type 2 diabetes. Diabetes care, 2002. 25(3): p. 458-463. DOI: $10.2337 /$ diacare.25.3.458

12. Sarfo, J.O. and M.-K. C.C., Type 2 Diabetes Mellitus, Depression and Neuropsychological Profiles Among Adults in Ghana. European Journal of Medicine, 2014. 1(1): p. 44-51. DOI: 10.13187/ejm.s.b.2014.1.44

13. de Queiroz, F.A., A.E. Pace, and C.B. dos Santos, Cross-cultural adaptation and validation of the instrument diabetes-39 (d-39): Brazilian version for type 2 diabetes mellitus patients-stage 11 . Revista latino-americana de enfermagem, 2009. 17(5): p. 708-715. DOI: $\underline{10.1590 / \mathrm{s} 0104-11692009000500018}$

14. KDOQI, Clinical Practice Guidelines and Clinical Practice Recommendations for Anemia in Chronic Kidney Disease. Am.J.Kidney Dis, 2006. 47(S11-S145). DOI: 10.105 3/j.ajkd.2006.03.011

15. Levey, A.S., K.-U. Eckardt, Y. Tsukamoto, A. Levin, J. Coresh, J. Rossert, D.d. Zeeuw, T.H. Hostetter, N. Lameire, and G. Eknoyan, Definition and 
classification of chronic kidney disease: a position statement from Kidney Disease: Improving Global Outcomes (KDIGO). Kidney international, 2005. 67(6): p. 2089-2398.DOI:10.1111/j.1523-

$1755.2005 .0 \quad 0365 . \mathrm{x}$

16. Al Rubaish, A., L. Wosornu, and S.N. Dwivedi, Using Deductions from Assessment Studies towards Furtherance of the Academic Program: An Empirical Appraisal of Institutional Student Course Evaluation. 2011.

DOI: 10.4236/ib.2011.32029

17. Danquah, I., G. Bedu-Addo, K.-J. Terpe, F. Micah, Y.A. Amoako, Y.A. Awuku, E. Dietz, M. van der Giet, J. Spranger, and F.P. Mockenhaupt, Diabetes mellitus type 2 in urban Ghana: characteristics and associated factors. BMC Public Health, 2012. 12(1): p. 1. DOI: $10.1186 / 1471-$ 2458-12-210

18. Jacobson, A.M., M.D. Groot, and J.A. Samson, The Evaluation of Two Measures of Quality of Life in Patients With Type I and Type II Diabetes. Diabetes Care, 1994. 17(4): p. 267-274 DOI: 10.2337/diacare.17.4.267.

19. Glasgow, R.E., L. Ruggiero, E.G. Eakin, J. Dryfoos, and L. Chobanian, Quality of life and associated characteristics in a large national sample of adults with diabetes. Diabetes care, 1997. 20(4): p. 562-567. DOI: $10.2337 /$ diacare.20.4.562

20. D'Souza, M.S., R. Venkatesaperumal, S.D. Ruppert, S.N. Karkada, and D. Jacob, Health Related Quality of Life among Omani Men and Women with Type 2 Diabetes. Journal of Diabetes Research, 2016. 2016: p. 10 DOI: 10.1155/2016/8293579.

21. Pashos, C.L., C.R. Flowers, N.E. Kay, M. Weiss, N. Lamanna, C. Farber, S. Lerner, J. Sharman, D. Grinblatt, I.W. Flinn, M. Kozloff, A.S. Swern, T.K. Street, K.A. Sullivan, G. Harding, and Z.M. Khan, Association of health-related quality of life with gender in patients with B-cell chronic lymphocytic leukemia. Supportive Care in Cancer, 2013. 21(10): p. 2853-2860 DOI: 10.1007/s00520-0131854-Z.

22. Svenningsson, I., C. Björkelund, B. Marklund, and B. Gedda, Anxiety and depression in obese and normal-weight individuals with diabetes type 2: A gender perspective. Scandinavian journal of caring sciences, 2012. 26(2): p. 349-
354. DOI: $10.1111 / \mathrm{j} .1471-6712$. 2011.00940.x

23. Vinik, A.I., R.E. Maser, B.D. Mitchell, and R. Freeman, Diabetic Autonomic Neuropathy. Diabetes Care, 2003. 26(5): p. 1553-1579 DOI: $\underline{10.2337 /}$ diacare.26.5.1553.

24. Mehdi, U. and R.D. Toto, Anemia, Diabetes, and Chronic Kidney Disease. Diabetes Care, 2009. 32(7): p. 13201326 DOI: $10.2337 / \mathrm{dc} 08-0779$.

25. Eghan, B., M.T. Frempong, and M. Adjei-Poku, Prevalence and predictors of microalbuminuria in patients with diabetes mellitus: a cross-sectional observational study in Kumasi, Ghana. Ethnicity and Disease, 2007. 17(4): p. 726.

26. Amoako, Y.A., D.O. Laryea, G. BeduAddo, H. Andoh, and Y.A. Awuku, Clinical and demographic characteristics of chronic kidney disease patients in a tertiary facility in Ghana. The Pan African Medical Journal, 2014. 18. DOI : 10.116 04/ pamj.2014.18.274.4192

27. Ephraim, R.K.D., S. Biekpe, S.A. Sakyi, P. Adoba, H. Agbodjakey, and E.O. Antoh, Prevalence of chronic kidney disease among the high risk population in South-Western Ghana; a cross sectional study. Canadian Journal of Kidney Health and Disease, 2015. 2: p. 40 DOI: 10.1186/s40697-015-0076-3.

28. Huang, E.S., S.E.S. Brown, B.G. Ewigman, E.C. Foley, and D.O. Meltzer, Patient Perceptions of Quality of Life With Diabetes-Related Complications and Treatments. Diabetes Care, 2007. 30(10): p. 2478-2483 DOI: 10.2337/dc07-0499.

29. Solli, O., K. Stavem, and I. Kristiansen, Health-related quality of life in diabetes: The associations of complications with EQ-5D scores. Health and Quality of Life Outcomes, 2010. 8(1): p. 1-8 DOI: 10.1186/1477-7525-8-18.

30. Parkerson Jr, G.R., R.T. Connis, W.E. Broadhead, D.L. Patrick, T.R. Taylor, and J.T. Chu-Kit, Disease-specific versus generic measurement of health-related quality of life in insulin-dependent diabetic patients. Medical care, 1993. 31(7): p. 629-639. DOI: 10.1097/00005650-199307000-00005

31. Sarfo, J.O., J. Cudjoe, and M.C. Schlatter, Health-Related Quality of Life Indicators in Ghana: Comparing Type 2 
Diabetic and Control Groups Journal of Advocacy, Research and Education, 2015. 3(2).

32. Yee, J., Diabetic Kidney Disease: Chronic Kidney Disease and Diabetes. Diabetes Spectrum, 2008. 21(1): p. 8-10 DOI: $10.2337 /$ diaspect.21.1.8.

33. Mosaku, K., K. Babatope, and R. Ikem, Depression, anxiety and quality of life among diabetic patients: a comparative study. Journal of the national medical association, 2008. 100(1): p. 73. DOI: 10.1016/s0027-9684(15)31178-0

34. Rubin, R.R. and M. Peyrot, Quality of life and diabetes. Diabetes/metabolism research and reviews, 1999. 15(3): p.
205-218. DOI: 10.1002/(SICI)1520-75

60(199905/06)15:3

35. Testa, M.A. and D.C. Simonson, Health economic benefits and quality of life during improved glycemic control in patients with type 2 diabetes mellitus: a randomized, controlled, double-blind trial. Jama, 1998. 280(17): p. 1490-1496. DOI: $10.1001 /$ jama.280.17.1490

36. Cade, W.T., Diabetes-Related Microvascular and Macrovascular Diseases in the Physical Therapy Setting. Physical Therapy, 2008. 88(11): p.13221335. DOI: $10.2522 / \mathrm{ptj} .20080008$. 